\title{
Security of Cloud Computing Using Adaptive Neural Fuzzy Inference System
}

\author{
Shumaila Shahzadi, Bushra Khaliq, Muhammad Rizwan (iD, and Fahad Ahmad \\ Department of Computer Science, Kinnaird College for Women, Lahore, Pakistan \\ Correspondence should be addressed to Muhammad Rizwan; muhammad.rizwan@kinnaird.edu.pk
}

Received 30 December 2019; Accepted 18 January 2020; Published 27 February 2020

Academic Editor: Angel M. Del Rey

Copyright (C) 2020 Shumaila Shahzadi et al. This is an open access article distributed under the Creative Commons Attribution License, which permits unrestricted use, distribution, and reproduction in any medium, provided the original work is properly cited.

\begin{abstract}
Cloud computing can enable organizations to do more by breaking the physical bonds between an IT foundation. The raised security dangers in cloud computing must be overpowered to profit the new processing perspective that offers an imaginative arrangement of activity for relationship to IT. The purpose of the study was to reduce security's obstacles and risks by using protection methods and approaches to ensure maximum data protection, which allows for the user to select the original security level. An adaptive neural control fuzzy system is used to resolve the unsecure and risky tasks of cloud computing. Sugeno control methods have been applied for these data protection issues in which the uncertainty because of randomness can be resolved. ANFIS identified the input parameters according to the current scenario, fuzzified the data, and integrated them into knowledge rule base. Different membership functions were used for training the data. In this article, we present a point-by-point examination of the cloud security issue. We assessed the issue from the cloud building point of view. In context of this examination, we deduce an unmistakable detail of the cloud security issue and key highlights that ought to be confirmed by any proposed security strategy. The examination and results show that the parameters dependent on ANFIS are very much intended to distinguish the oddities in cloud condition with least bogus negative rate and high discovery precision. The performance of Sugeno membership function usually gives better results and ensures the computational efficiency and accuracy of data.
\end{abstract}

\section{Introduction}

Cloud computing in its many forms has proven to be an amazing, persuasive arrangement of advancements, which can furnish even the littlest undertaking with the noteworthy advantages. Cloud-based frameworks engage this adaptability by empowering simple and consistent access to basic documents from any area or gadget. They take into consideration straightforward document and data sharing, just as enhanced correspondence [1].

Worldwide distributed storage foundation additionally enables organizations to expand their compass, quicken time, and offer its items around the world. It mitigates the expenses of on location information stockpiling, enhances excess, and limits overhead [2]. The expanding interest for distributed computing has turned into the pattern of associations with the expectation that they can convey snappy, simple, and adaptable administrations. Be that as it may, it additionally raises a few concerns, particularly when associations are excessively excited and fast in settling on choices without distinguishing and thinking about what the association needs. One of the worries is identified with understanding the contrasts between customary condition and distributed computing condition, which manage the qualities of some computational assets, for example, programming, stage, and different frameworks, including different attributes. Every association will require diverse computational assets. This was affected by the business perspective of the association, which has specific qualities not the same as the attributes of different associations, notwithstanding when they have a place with a similar sort of association. Or maybe, they have a short life time and turn into a weight for the associations [3]. 
The key systems which are proposed to defeat the issues include

(a) compositional view, that is a comprehension of the design measurement, which uncovers the necessities of computational assets dependent on business see; (b) an arrangement see, that is a mix and institutionalization show ready to give point by point depictions and mapping of the requirements; and (c) the versatile view, that is a plan of parameter needs and capacities to adaptively understand the required administrations.

We regularly locate that numerous undertakings are as of now overseeing around five to 16 distinctive cloud sellers, which can present new dangers and vulnerabilities because of the absence of predictable administration, control, and deceivability into the risk stance of uses and information. To help defeat these difficulties, engineers coordinate security as priority into the application improvement process. Consolidating security best practices into each feature of utilization plan and organization will give more prominent application deceivability, control, and assurance.

1.1. Problem Discussed. Regardless of the potential preferences and salaries that are obtained from the circulated registering model, the model still has a huge amount of open issues that influence the model significance and certainty. The mostly researched area about the computing model are seller lock-in, multi-occupancy and segregation, information on the board, administration compactness, flexibility motors, SLA (service level agreement) of the board, and cloud. From the cloud purchasers' point of view, security is the significant worry that hampers the reception of the distributed computing modeling light of the fact that:

(i) Enterprises reappropriate the security rights to an administrator that has their IT resources.

(ii) Copresence of the advantages of various occupants in a similar area and utilizing a similar example of the administration while being uninformed of the quality of security controls utilized.

(iii) The absence of security ensures in the SLAs between the cloud buyers and the cloud suppliers.

(iv) Hosting, this arrangement of significant resources on freely accessible framework builds the likelihood of assaults.

From the cloud suppliers' point of view, security requires a great deal (security arrangements' licenses), assets (security is an asset expending undertaking), and is a troublesome issue to ace (as we talk about later). Regardless, skipping security from the disseminated processing model guide will harm ordinary salaries as illuminated beforehand. So, cloud providers need to understand purchasers' stresses and pursuit out new security courses of action that settle such concerns [4].

1.2. Motivation. In this article, we break down existing difficulties and issues engaged with the distributed computing security issue. We assemble these issues into engineering-related issues, administration conveyance-related issues, cloud trademark-related issues, and cloud partner-related issues $[5,6]$. Our goal is to distinguish the powerless focuses in the cloud show.

We present a point-by-point examination for every shortcoming to feature their underlying drivers. This will help cloud suppliers and security sellers to have a superior comprehension of the issue. It additionally helps scientists monitoring the current issue measurements and holes.

1.3. Contribution. This study is the investigation of information security systems utilized for ensuring and verifying information in cloud all through the world. It talks about the potential dangers to information in the cloud and their answers embraced by different specialist organizations to defend information.

Various security aspects such as for data loss, data integrity, shared environment, data breaches, and insecure application programming delineate a few wanted arrangements that should be accessible in cloud-based applications:

(i) Secure correspondence.

(ii) Internal Data insurance.

(iii) Denial of administration and vitality squander.

(iv) Depersonalization of information however keeping the specific situation.

Organization: The rest of this article is organized as follows. In Section 2, the literature is reviewed. Section 3 security issues are discussed. Section 4 proposes the principles and the fundamentals for securing the cloud computing. Section 5 is a simulation of cloud computing. Section 6 discusses and concludes the research. Section 7 concludes the research with some future challenges.

\section{Literature Review}

Soni et al. [1] presented Security and privacy in cloud computing in which they analyses the key security issues that envelopes market today with safety measures, to serve server providers and enterprises in the best way possible.

El-Yahyaoui and Dafir [3] presented Data privacy in cloud computing, they present a new fully homomorphic encryption scheme from integers. Our encryption scheme can be used essentially to secure sensible data in cloud computing. The proposed scheme uses a large integer ring as clear text space and one key for encryption and decryption, i.e., it is a symmetric encryption scheme.

So as to comprehend the rudiments of distributed computing and putting away information verifying on the cloud, a few assets have been counseled. This area gives a survey of writing to set an establishment of talking about different information security perspectives. In [7] the authors give phenomenal understanding into the fundamental ideas of distributed computing. A couple of key thoughts are explored in this paper by giving cases of usages that can be made using dispersed registering and how they can help the making scene in getting benefit by this rising development. 
Devi and Ganesan [8] presented Environmental Benefits of Enhanced Hecc-Elgamal Cryptosystem for Security in Cloud Data Storage Using Soft Computing Techniques. Notwithstanding present methods used for encrypting the files in cloud they are not highly efficient. The authentication of the user is verified successfully dual encryption is performed on the cloud stored files using ElGamal cryptosystem and Hyper Elliptical Curve Cryptography (HECC).

Existing security systems does not consider the narrowmindedness related with the portable hubs in the MANET. Moreover, the current plans additionally accept that there is a focal foundation for validation purposes, which isn't the situation for specially appointed systems [9-12].

Imran Tariq [13] presented Agent Based Information Security Framework for Hybrid Cloud Computing. This paper proposes Agent Based Information Security Framework for Hybrid Cloud Computing as an all-inclusive method including cloud related methods.

A gander at the security issue by ensuring data control to the end customer to flood assurance represented by Tjoa, A. M. A couple of Cloud considering ambushes are looked along with and a couple of plans are proposed to vanquish these strikes. Thus, Mohamed et al. [14] proposed a data security appear for circulated processing reliant on cloud designing. They in like manner made programming to propel the effort in Data Security show for conveyed figuring further.

Cayirci et al. [15] presented Cloud Adoption Risk Assessment Model which is designed for cloud customers to assess the risks that they face by selecting a specific cloud service provider. It is an expert system to evaluate various background information obtained from cloud customers, cloud service providers and other public external sources, and to analyze various risk scenarios. This would facilitate cloud customers in making informed decision to select the cloud service provider with the most preferable risk profile.

A considerable lot of the current proposition in the zone of secure versatile frameworks for remote impromptu system address the issue of host assurance against pernicious specialists. So far, a little research is done on shielding a versatile operator from malevolent hosts or egotistical hubs. The mystery of both specialist's vital and code/state parts speaks to a testing issue.

\section{Security Challenges}

Distributed computing security difficulties and issues talked about different analysts. The Cloud Computing Use Cases amass talks about the distinctive use case situation necessities that may exist in the distributed computing model.

They consider use cases from alternate points of view including clients, designers, and security engineers explored the diverse security dangers identified with receiving distributed computing alongside the influenced resources, the danger probability, effects, and vulnerabilities in distributed computing that may prompt such dangers.

The accompanying rundown contains a few security issues difficulties: (i) In "Threats to Cloud Computing" by ANFIS examine the security details on the data loss, data integrity, shared environment, data breaches, and insecure application programming identified with information areas, isolation, information stockpiling, and information recuperation.

(ii) High-dimension security distributed computing model, for example, information honesty, installment, and protection of touchy data.

(iii) One of the most genuine dangers to distributed computing itself originates from HTTP Denial of Service or X ML-Based Denial of Service assaults. These sorts of assaults are straightforward and simple to actualize by the aggressor, yet to security specialists they are twice as hard to stop.

(iv) Security vulnerabilities existing in the cloud stage.

(v) Security challenges administration conveyance show, concentrating on the data loss, data integrity, shared environment, data breaches, and insecure application programming. ANFIS talks about basic regions of distributed computing $[11-13,16]$. They convey a lot of best practices for the cloud supplier, customers, and security sellers to follow in every space. ANFIS point-by-point reports examining for a portion of these areas. To help us understand the issues and arrangements, a profound examination in the cloud model ought to be performed to distinguish the main drivers and key taking an interest measurement in such security issues/issues.

(a) Rightness: the capacity to play out a particular arrangement of required tasks.

(b) Strength: the capacity of the framework to keep up its capacities under startling and surprising circumstances that can happen inside or remotely.

(c) Extensibility: the capacity of the smart control to help arranged and impromptu overhauls (equipment or programming) without the requirement for replan.

(d) Reusability and similarity: the capacity to utilize subframeworks and segments in various applications and similarity with new circumstances. There are three fundamental strategies for wise control, two of which are fluffy rationale and neural systems.

\section{Protecting Data Using Adaptive Neural Fuzzy Interference System}

Before we talk about our model further, it is basic to clarify what we mean by versatile security and all-encompassing security. We characterize versatile security as a conglomeration of conventional safety efforts, helplessness checking, helplessness recognition, and weakness reaction in a specific order [17-19].

By versatile security we mean the security demonstrate that responds what's more, and alters the security of the 
framework dependent on required security level or powerlessness level. By all encompassing security we mean proactive, preventive, and prescient security demonstrate, which can be available to change, yet endeavors to determine the issue of assaults by preventive innovation. The main dimension of insurance in such engineering is in view of the partition between client-to-cloud and device-to-cloud information trade. This will altogether lessen the low dimension assaults on the gadgets which is considered as one of the fundamental issues. The second basic increases are the utilization of firewall at cloud connector door and each passageway purpose of the cloud. The third thing is keeping the gadget organize private and secure-validation and encoded messages ought to be $[20,21]$.

What's more, the last mainstay of secure engineering is the improvement of correspondence channels-physical gadgets will match just with its virtual cases. Therefore, it will be simpler to screen correspondence and give trust.

4.1. Neural System. Control engineers see neural frameworks as huge-scale, nonstraight incredible systems that are described inside a first demand differential condition. Neural frameworks are in fact new structures for information planning systems that contain different associated dealing with parts. The associations that interface these getting ready parts are called interconnections. Each dealing with part has somewhere around one wellsprings of data and a lone yield. Every data have a weight allotted to them, and these heap vectors change by learning rules. There can be an issue of how such a huge unique framework forms their data? The response to this inquiry is the utilization of vitality capacities for the framework. Each nondirect powerful framework has a few balance focuses. These focuses are the base focuses on the vitality scene. In the event that a discretionary information design is given to the framework as its underlying state, the framework is fit for moving toward one of these harmonies directs subject toward the worldwide soundness of the framework [22-27].

Figure 1 elaborates the neural system of the distributed computing security issues.

4.2. Architecture of Fuzzy Logic Controllers. Neuro-Fuzzy Systems Based on Intelligent Control Fundamental setup of fluffy rationale controllers.

Fuzzification interface which comprises the following advances:

(1) Estimating the estimation of info factors.

(2) Exchange of a wide scope of information variable qualities to the related general set.

(3) Fuzzification, which is in certainty the discussion of information to appropriate phonetic esteem.

(4) Knowledge base, including "information base" and "phonetic (fluffy) control rule base."

(5) The information base gives the important definitions that are used to characterize phonetic control rules and fluffy information.

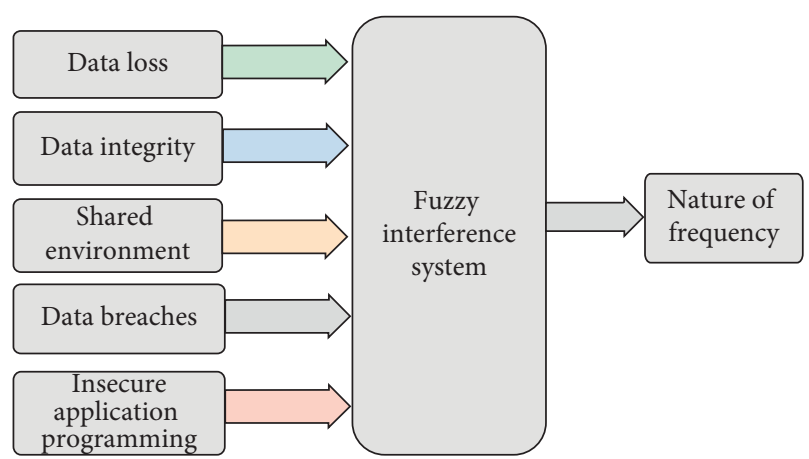

FIgURE 1: Adaptive fuzzy interference system.

(6) Estimating the estimation of info factors.

(7) Exchange of a wide scope of information variable qualities to the related general set.

(8) Fuzzification, which is in certainty the discussion of information to appropriate phonetic esteem.

(9) Knowledge base, including "information base" and "phonetic (fluffy) control rule base."

(10) The information base gives the important definitions that are used to characterize phonetic control rules and fluffy information.

(11) The standard base, which determines the control objectives utilizing a set of semantic control rules.

(12) Decision-making rationale, which is the bit of the fluffy rationale controller. In this part, human basic leadership is reproduced dependent on fluffy ideas and inducing fluffy control activities by utilizing fluffy ramifications and tenets of deduction.

(13) Defuzzification interface.

(14) Change of a wide scope of yield factors to the allinclusive set.

(15) Defuzzification of principles and etymological factors as it were that is retrievable and reasonable by whatever is left of process.

The general correspondence between a client and a gadget ought to be constrained to the association with the virtual occurrence utilizing the fitting security shown in Figure 2. In the event that a solid encryption is required, it will be less demanding to actualize on the virtual gadget in the cloud than on the low-asset physical gadget. Expelling the alternate ways from client to physical gadgets will decrease altogether the security necessities for the engineering and will decrease the unpredictability.

Implementation of an algorithm.

(1) Parameter estimation:

The algorithm uses these parameters

(i) data loss

(ii) data integrity

(iii) shared environment

(iv) data breaches

(v) insecure application programming 


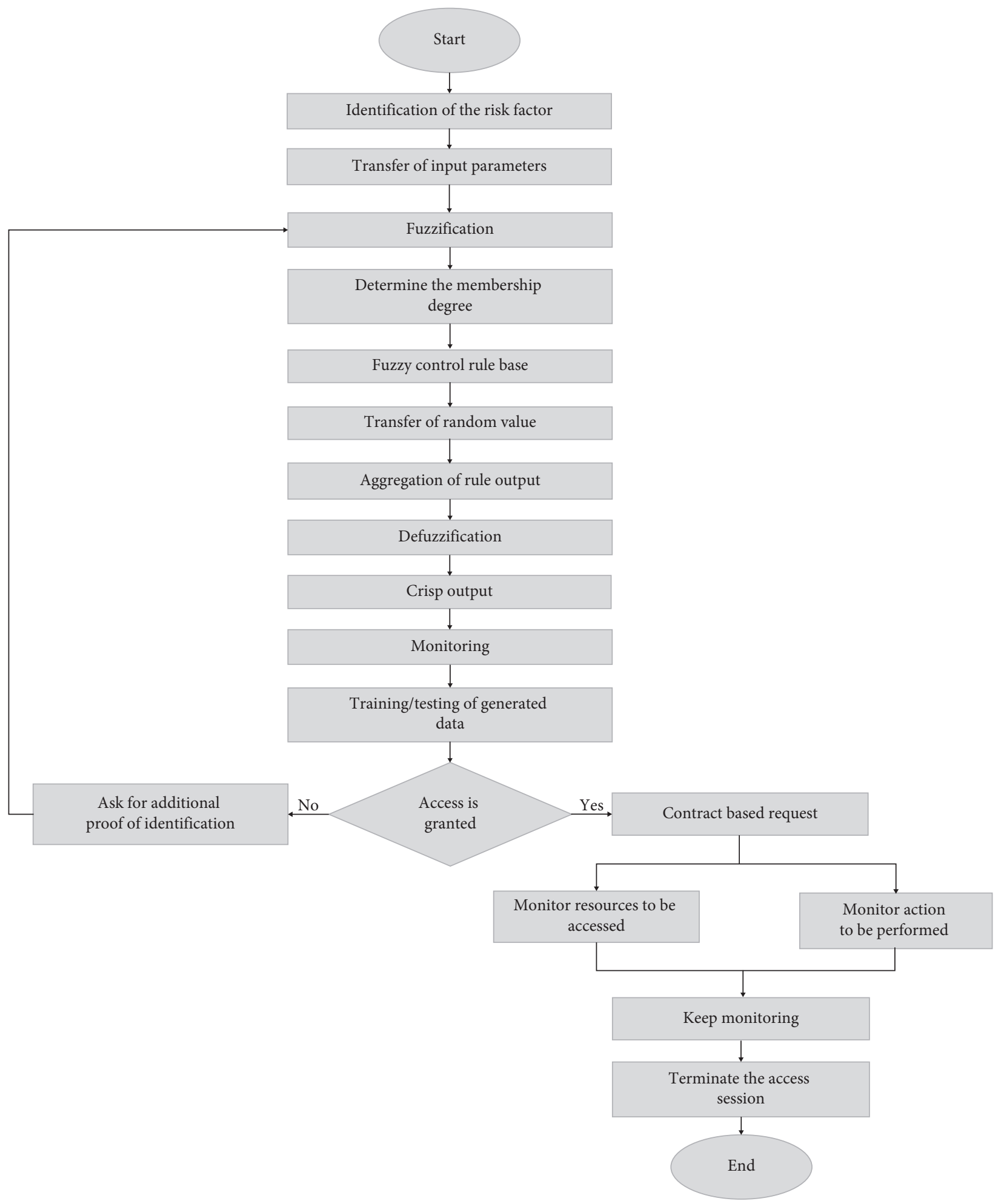

FIGURE 2: Flowchart of adaptive control system.

(2) Each parameter has a linguistic label.

(3) Generate the membership function for input and output structures.

(4) Each parameter generates membership grades using membership functions.
(5) The aggregate output is the product of all input signals and represents the strength of a rule, usually AND operator is applied to compute the incoming signal.

(6) Compute the overall output. 
(7) Train the data.

(8) ANFIS parameters are trained/updated.

(9) Generate and analyze errors.

The utilization could be in advantage of security assurance as various could be doled out to one physical gadget that give just the information that is proper for the application, which will confine the opportunity of information break and benefit taking. Every client cloud has its own perspective on the gadget administrations and parameters actualizing sensor-as-an administration or sensing-as-an administration worldview.

Regardless of what security conventions and advancements are connected, the accepted procedures in complex security frameworks as cloud are depending on standard checking and defenselessness checks. The issue is that in such complex frameworks, observing the security is a Big Data undertaking, which is intricately independent from anyone else.

\section{Simulation}

Among various blends of approaches in sensitive figuring, the one that has the most raised detectable quality starting at now is that of soft justification and neuro computing, inciting the alleged neuro-fuzzy systems. A convincing system for this requirement is ANFIS Sugeno model shown in Figure 3. The ANFIS structure is a class of flexible framework that uses Sugeno Fuzzy Inference Systems. There are some showing conditions in data perceive the Membership Functions (MFs) ought to take after. The parameters related with a given MF self-confidently could be picked to a degree that leads to the yield data to speak to these sorts of assortments in the data regards. So, the alleged neuro-flexible learning framework united into ANFIS.

Accept a fluffy derivation framework in which five sources of input and one yield. Fluffy set with two fluffy on the off chance that rules are as per the following:

On the off chance that $x$ is $\mathrm{A} 1$ and $y$ is $\mathrm{B} 1$, at that point $f 1=p 1 x+q 1 y+r 1$.

On the off chance that $x$ is A2 and $y$ is B2, at that point $f 2=p 2 x+q 2 y+r 2$.

We can execute the reasoning instrument into a feed forward neural framework with oversaw learning capacity, which is known as the ANFIS structure.

ANFIS gives a methodology to the soft showing system to learn information about a dataset, to process the MF parameters, represented in Figure 4 that best empower the related fleecy determination structure to pursue the given data/yield data. This learning strategy works additionally, to that of neural frameworks.

Membership functions (MFs) are the building blocks of fuzzy set theory; that is, fuzziness in a fuzzy set is determined by its MF. Accordingly, the shapes of MFs are important for a particular problem because they effect on a fuzzy inference system. They may have different shapes such as triangular, trapezoidal, and Gaussian. In this article, triangular fuzziness is used because triangular shapes represent fuzzy numbers. Triangular Fuzzy Sets

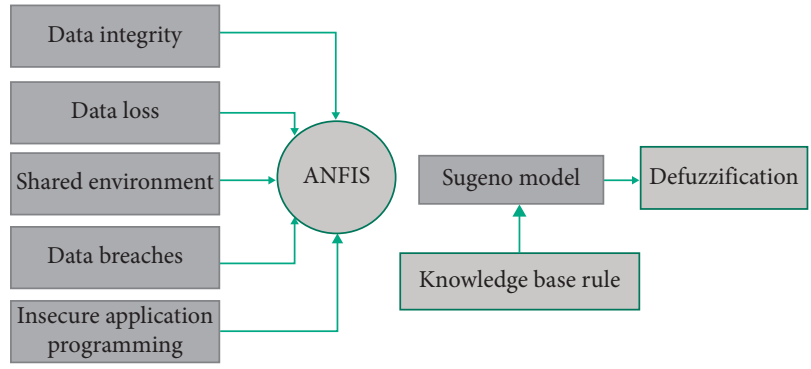

Figure 3: Sugeno model.

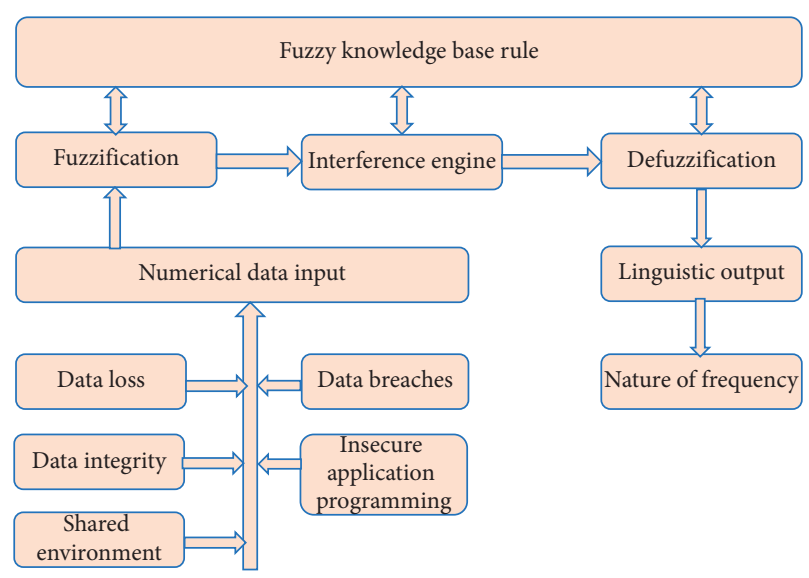

Figure 4: Fuzzy System model.

$$
f(x)= \begin{cases}\frac{(x-a)}{(b-a)}, & a<x<b, \\ \frac{(c-a)}{(c-b)}, & x \geq 0 .\end{cases}
$$

The parameters related with the MFs will change through the learning strategy. ANFIS uses either back expansion or a mix of least square estimations and back causing for $\mathrm{MF}$ parameter estimations. The per users are insinuated for more nuances on these methods.

Before we start the ANFIS setting, we need to deliver our Fuzzy Inference System (FIS). FIS can complete in network distributing subtractive gathering. In lattice partitioning, all the possible standards are created reliant on the amount of MFs for every information. For example, in a two-dimensional data space, with three MFs in the data sets, the number of rules in system distributing results in various fundamentals represent in Figure 5. This allocating need only couple of MFs for every information, and it encounters issues when we have a honorably gigantic number of information sources.

By then, ANFIS is associated for further modifying of the MFs.

The different MFs which are used for inputs are

for data loss, MF: (no data loss, medium data loss, and full data loss), for data integrity, MF: (no data integrity, medium data integrity, and high data integrity), for shared environment, MF: (no shared environment, medium shared 


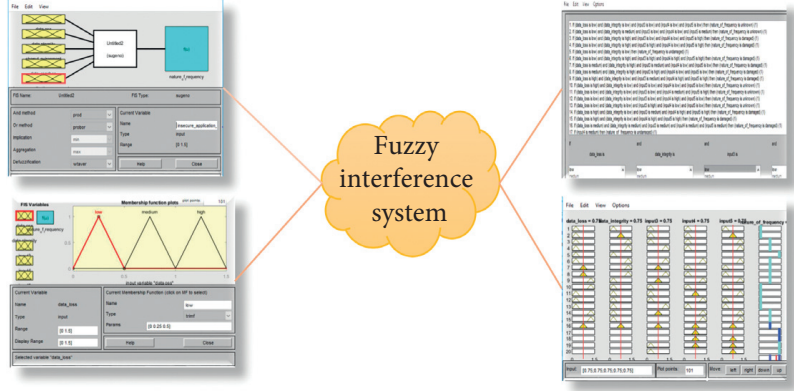

FIgURe 5: Fuzzy interference system.

environment, and high shared environment), for data breaches, MF: (no data breaches, medium data breaches, and high data breaches), and for insecure application programming, MF: (no insecure application programming, medium insecure application programming, and high insecure application programming).

Membership functions are mathematically described as

$$
m A(x)=\{1 x € A, 0 x € A\} m A(x) €\{0,1\} .
$$

It comprises various member function; fluffy guidelines and the standards are put away in the database. The procedure obviously expounds the means included in the structuring of enrollment capacities and the principles from these participation capacities. The aftereffects of the model are investigated, and a choice is made after the conclusive outcomes. In the last advance endorsement of the most secure part ought to be taken from the skilled position and the most secure segment is conveyed to the fashioner of the framework.

Figure 6 shows the different membership functions that are as follows: no (data loss), medium (data loss), and full (data loss) for the input labeled as data loss. The degree of membership functions is plotted as follows: no (data loss) is between 0 and 1 , medium (data loss) is $1-2$, and full (data loss) is $2-3$.

Figure 7 shows the different membership functions that are as follows: no (data integrity), medium (data integrity), and full (data integrity) for the input labeled as authentication. The degree of membership functions is plotted as follows: no (data integrity) is between 1 and 1.667, medium (data integrity) is $1.667-2.333$, and full (data integrity) is 2.333-3.

Figure 8 shows different membership functions that are as follows: no (shared environment), medium (shared environment), and full (shared environment) for the input labeled as nonrepudiation. The degree of membership functions is plotted as follows: no (shared environment) is between 1 and 1.667, medium (shared environment) is 1.667-2.333, and full (shared environment) is 2.333-3.

Figure 9 shows the different membership functions that are as follows: no (data breaches), medium (data breaches), and high (data breaches) for the input labeled as data confidentiality. The degree of membership functions is plotted as follows: no (data breaches) is between 0 and 0.5 ,

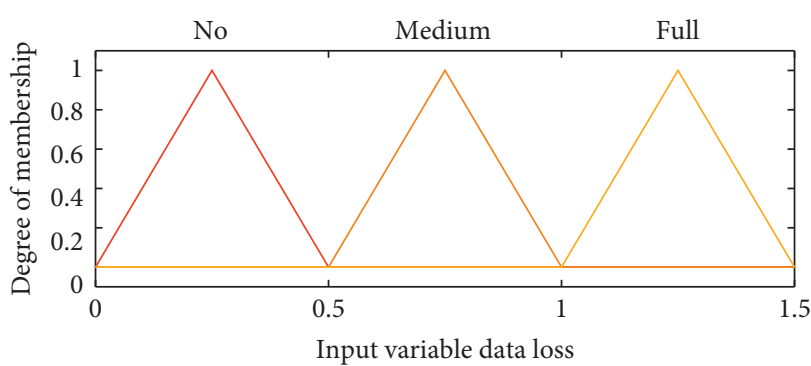

FIgURE 6: Input variable data loss.

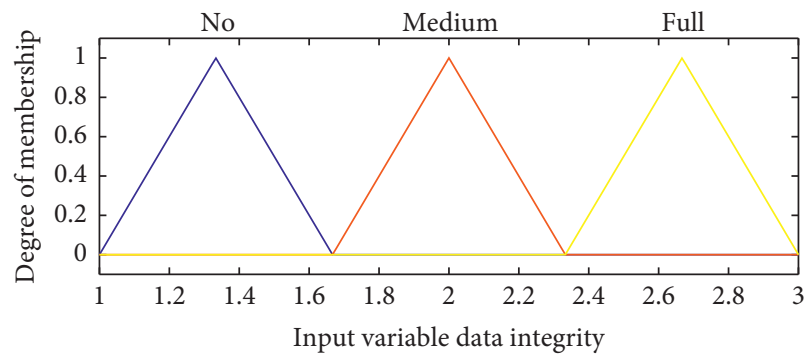

FIgURE 7: Input variable data integrity.

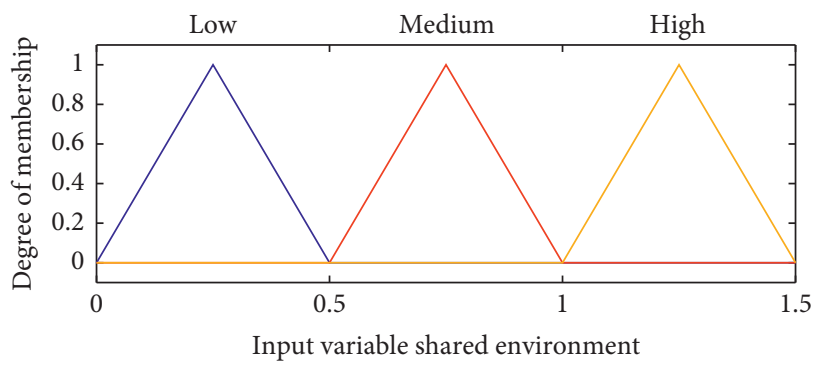

FIGURE 8: Input variable shared environment.

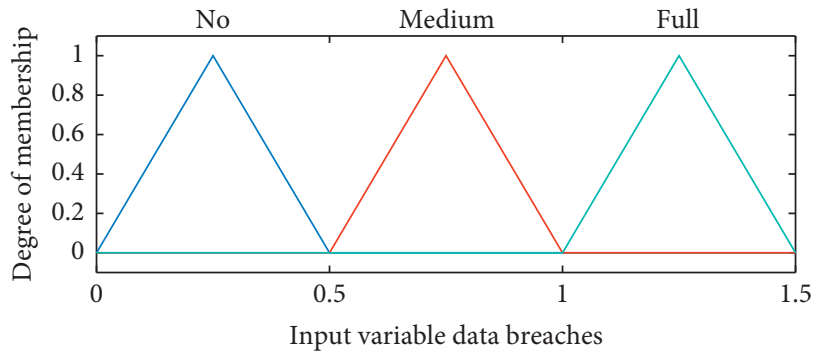

Figure 9: Input variable data breaches.

medium (data breaches) is $0.5-1$, and full (data breaches) is $1-1.5$.

Figure 10 shows the different membership functions that are as follows: no (insecure application programming), medium (insecure application programming), and high (insecure application programming) for the input labeled as insecure application programming. The degree of membership functions is plotted as follows: no (insecure application programming) is between 0 and 1 , medium (insecure 


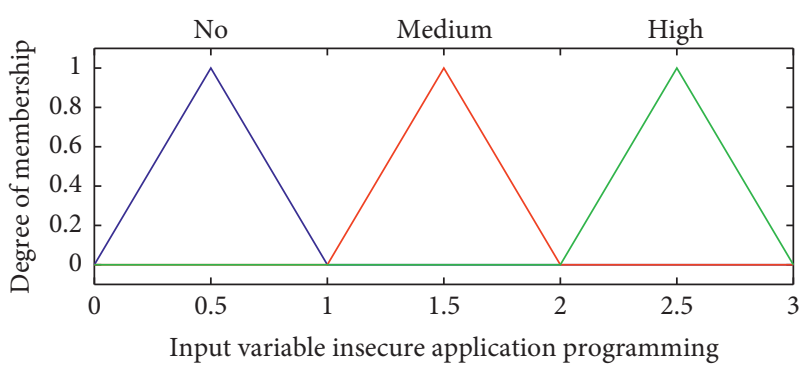

FIgURE 10: Input variable insecure application programming.

application programming) is $1-2$, and high (insecure application programming) is $2-3$.

5.1. Security Attributes. The proposed strategy is applied to plan a model which assesses the security of segments as indicated by the accompanying particulars.

5.1.1. Data Loss. The information misfortune characteristic of security checks a blunder condition in data frameworks in which data are crushed by disappointments or disregard away, transmission, or preparing. The range of MF for data loss is given below

$$
\begin{aligned}
\mu(\text { data loss })=\{ & \{0<x<1=\text { no, } 1<x \\
& <2=\text { medium }, 2<x<3=\text { full }\} .
\end{aligned}
$$

5.1.2. Data Integrity. Information trustworthiness gives exactness and rightness of the data identified with programming parts. All information and data of the segments are shielded from unapproved changes, creation, and replication. Information honesty is numerically appeared underneath utilizing the condition:

Mathematically, it can be shown as follows:

$$
\begin{aligned}
& \mu(\text { data integrity })=\{1<x<1.667=\text { no, } 1.667<x \\
&<2.333=\text { medium, } 2.333<x<3=\text { full }\} .
\end{aligned}
$$

5.1.3. Shared Environment. System security makes a safe situation for clients, PCs and different gadgets, and interior systems. System security is a particular field inside PC organizing. It likewise varies from data security in that data security covers all types of data past computerized information. This aides in affirming the accessibility of information to an outsider as a proof that a few occasions have occurred.

$$
\begin{aligned}
\mu(\text { shared environment })=\{ & \{1<x<1.667=\text { no, } 1.667<x \\
& <2.333=\text { medium, } 2.333<x \\
& <3=\text { full }\} .
\end{aligned}
$$

5.1.4. Data Breaches. This characteristic of security shields information joined with programming parts from illicit or unapproved get to. A few information privacy strategies and calculations are utilized to verify information of accessible programming parts. MF for safety is given below in equation:

$$
\begin{aligned}
& \mu(\text { data breaches })=\{0<x<0.5=\text { no, } 0.5<x<1=\text { medium, } \\
& 1<x<1.5=\text { full }\} \text {. }
\end{aligned}
$$

5.1.5. Insecure Application Programming. Correspondence stream affirms that sharing of data identified with various programming parts is made uniquely with approved people. The segments are shielded from unlawful access.

$\mu$ (in secure application programming $)=\{0<x<1=$ no, 1

$$
\begin{aligned}
& <x<2=\text { medium, } \\
& 2<x<3=\text { full }\} .
\end{aligned}
$$

5.2. Design of Fuzzy Inference System. The proposed model is structured utilizing the fluffy tool kit. It comprises five fundamental GUI instruments including FIS manager, enrollment work supervisor, rule editorial manager, rule watcher, and surface watcher. The proposed model utilized the Sugeno fluffy deduction framework.

In view of the proposed strategy, the three participation capacities, and the five information sources, the fluffy guidelines are gotten.

These principles are in the structure as pursues:

(i) 1. If (data loss is no) and (data integrity is no) and (shared environment is low) and (data breaches are no) and (insecure_application_programming is no), then (nature_of_frequency is unknown) (1)

(ii) 2. If (data_loss is no) and (data_integrity is medium) and (shared_environment is no) and (data_breaches are no) and (insecure_application_programming is medium), then (nature_of_frequency is unknown) (1)

(iii) 3. If (data_loss is no) and (data_integrity is full) and (shared_environment is no) and (data_breaches are no) and (insecure_application_programming is high), then (nature_of_frequency is damaged) (1)

(iv) 8. If (data_loss is medium) and (data_integrity is full) and (shared_environment is high) and (data_breaches are no) and (insecure_application_programming is no), then (nature_of_frequency is damaged) (1)

(v) 12. If (data_loss is no) and (data_integrity is no) and (shared_environment is no) and (data_breaches are full) and (insecure_application_programming is no), then (nature_of_frequency is unknown) (1) and so on. 
Table 1 shows the fuzzy IF/THEN rules when output is undamaged. Table 2 shows the fuzzy IF/THEN rules when output is unknown, and Table 3 shows the fuzzy IF/THEN rules when output is damaged.

5.3. Fuzzy Inference System. A Sugeno display is created in Fuzzy Inference System by characterizing information misfortune, information respectability, shared condition, information ruptures, and unreliable application programming as framework inputs and the idea of recurrence as yield as appeared in Figure 11.

5.4. Membership Function Plot. The security string is taken as information and enrollment capacities are characterized by the appropriate ranges accordingly characterizing the dimension (low, medium, and high) as appeared in Figure 12.

5.5. Rule Viewer. Based on the on the off chance that rules characterized, a lot of qualities are obtained and handled utilizing rule watcher. The Rule Viewer exhibits the without hesitation perspective on the fluffy deduction framework's procedure. The Rule Viewer additionally delineates how the state of certain participation capacities impacts the last outcome. Each standard is a line of plots, and every section is a variable. The framework has a solitary yield, and got utilizing weighted normal defuzzification process, as appeared in Figure 13.

All yield participation capacities ought to be of comparable sort whether straight or a steady. In this examination study, steady sort participation capacities are utilized, as appeared in Figure 14.

5.6. Surface Viewer. After preparing and testing the handled information, a 3-D surface plot is acquired as appeared in Figure 15 with any two info factors on the event and vertical hub and the yield variable on the third hub. The surface watcher gives the office of analyzing it at various plots for any further amendments.

Figure 15 depicts the surface plot among the two input variables data loss and data integrity and output variable nature of frequency. We can see that input variable parameters have different effect on the output variable. As the nature of frequency increased with the increase in the data integrity and data loss is also at high peak.

Figure 16 shows that nature of frequency increases with the increases of data integrity and nature of frequency decreases with the decreases of data integrity. Hence, the simulation results clearly show that increases in data integrity provide best performance characteristics of monitoring access is not reliable.

Figure 17 shows that if data loss attribute at the highest peak than its effect on the nature of frequency. The data performance is not well secured.

Figure 18 shows that variation in insecure application programming effect the performance in the nature of frequency.
Figure 19 shows that as nature of frequency is secured it shows better performance if data breaches are low but if the probability of data integrity and data breaches increases, we need to monitor the data again.

All the 3D surface view results clearly show performance characteristics as compared with multiple attribute that we need to more authenticate the data or not in terms of network lifetime, stability, and throughput.

5.7. Neuro-Fuzzy Inference Model. The last neuro-fluffy deduction framework display is acquired as appeared in Figure 20, demonstrating the five information sources and a yield according to their distinctive blends.

5.8. ANFIS Testing and Training Performance. 50\% of the processed information is utilized for testing, 30\% of similar information is used for training, and $20 \%$ of information is used for checking reason utilizing hybrid calculation as appeared in Figures 21-23.

Model endorsement is the method by which the data vectors from information/yield educational lists on which the AFIS was not readied, are displayed to the readied AFIS model, to see how well the AFIS model predicts the relating enlightening record yield regards.

Figures 21-23 the testing informational index gives you a chance to check the speculation capacity of the subsequent fluffy deduction framework. The thought behind utilizing a checking informational index for model approval is that after a specific point in the preparation, the model starts over fitting the preparation informational index. On a fundamental level, the model blunder for the checking informational index will in general diminish as the preparation happens up to the point that over fitting starts, and afterward the model mistake for the checking information all of a sudden increment. Over fitting is represented by testing the FIS prepared on the preparation information against the checking information and picking the enrollment work parameters to be those related with the base checking blunder if these mistakes show model over fitting.

\section{Results and Discussion}

Based on the structured principles and model, the security of segments can be assessed. Table 4 shows input/output details and the membership functions used. Following is the configuration of the model as implemented in fuzzy logic.

$$
\begin{aligned}
& \text { Name = "cloud computing security model" } \\
& \text { Type = "sugeno" } \\
& \text { Num inputs = " } 5 " \\
& \text { Num outputs = " } 1 " \\
& \text { And method = "min" } \\
& \text { Or method = "max" } \\
& \text { Imp method = "min" } \\
& \text { Agg method = "max" } \\
& \text { Defuzz method = "centroid" }
\end{aligned}
$$


TABLE 1: Fuzzy IF/THEN rules when output is undamaged.

\begin{tabular}{lccccc}
\hline Data loss & Data integrity & Shared environment & Data breaches & Insecure application programming & Nature of frequency \\
\hline No & Low & No & No & Medium & Undamaged \\
No & Low & Medium & No & Medium & Undamaged \\
No & Medium & No & No & Medium & Undamaged \\
No & Medium & Medium & No & No & Undamaged \\
\hline
\end{tabular}

TABLe 2: Fuzzy IF/THEN rules when output is unknown.

\begin{tabular}{lccccc}
\hline Data loss & Data integrity & Shared environment & Data breaches & Insecure application programming & Nature of frequency \\
\hline Medium & Medium & Medium & No & No & Unknown \\
Medium & Medium & Medium & No & Medium & Unknown \\
Medium & Low & No & No & High & Unknown \\
No & Full & No & Medium & Medium & Unknown \\
\hline
\end{tabular}

TABle 3: Fuzzy IF/THEN rules when output is damaged.

\begin{tabular}{lccccc}
\hline Data loss & Data integrity & Shared environment & Data breaches & Insecure application programming & Nature of frequency \\
\hline No & Low & Full & Full & No & Damaged \\
Medium & Low & No & Medium & High & Damaged \\
Medium & Full & No & Full & Medium & Damaged \\
Full & Medium & No & No & Medium & Damaged \\
\hline
\end{tabular}

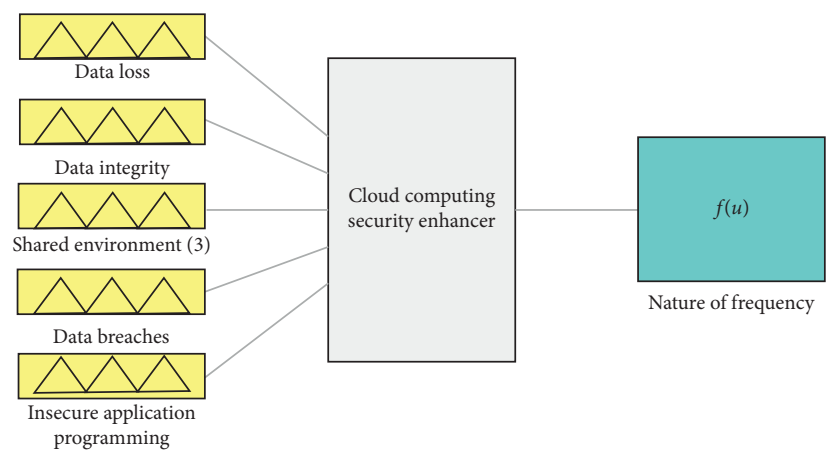

Figure 11: Sugeno model.

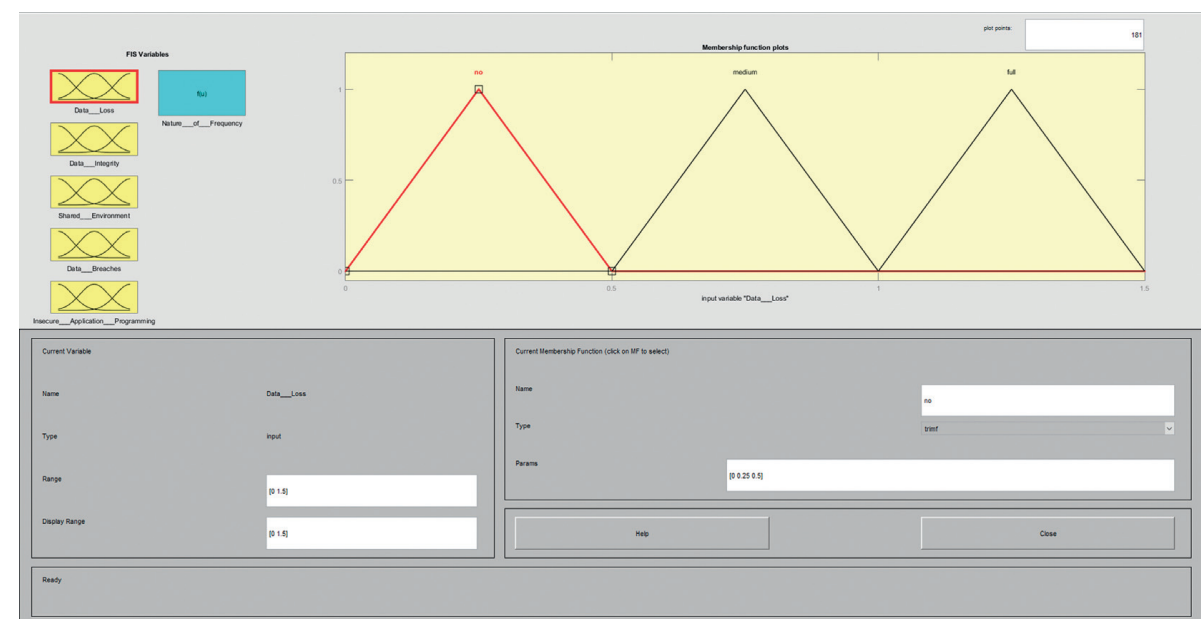

FIGURE 12: Membership functions correlating inputs with output. 


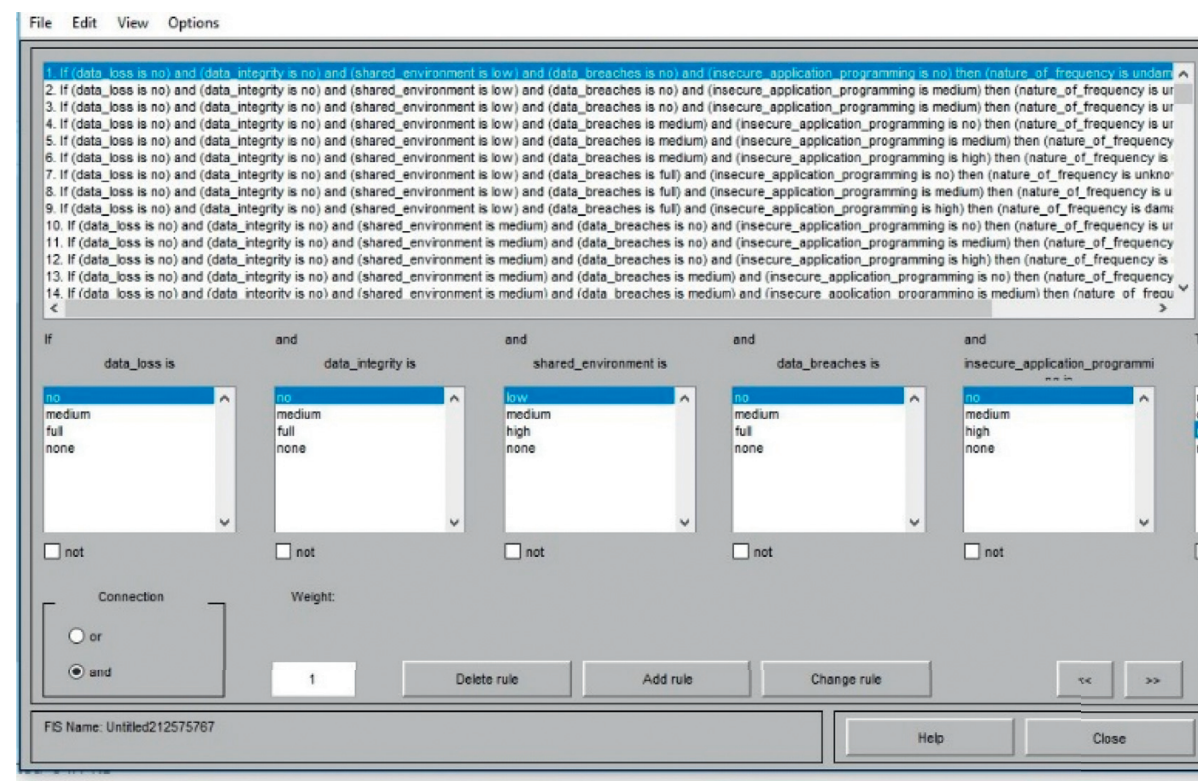

Figure 13: Fuzzy rule base.

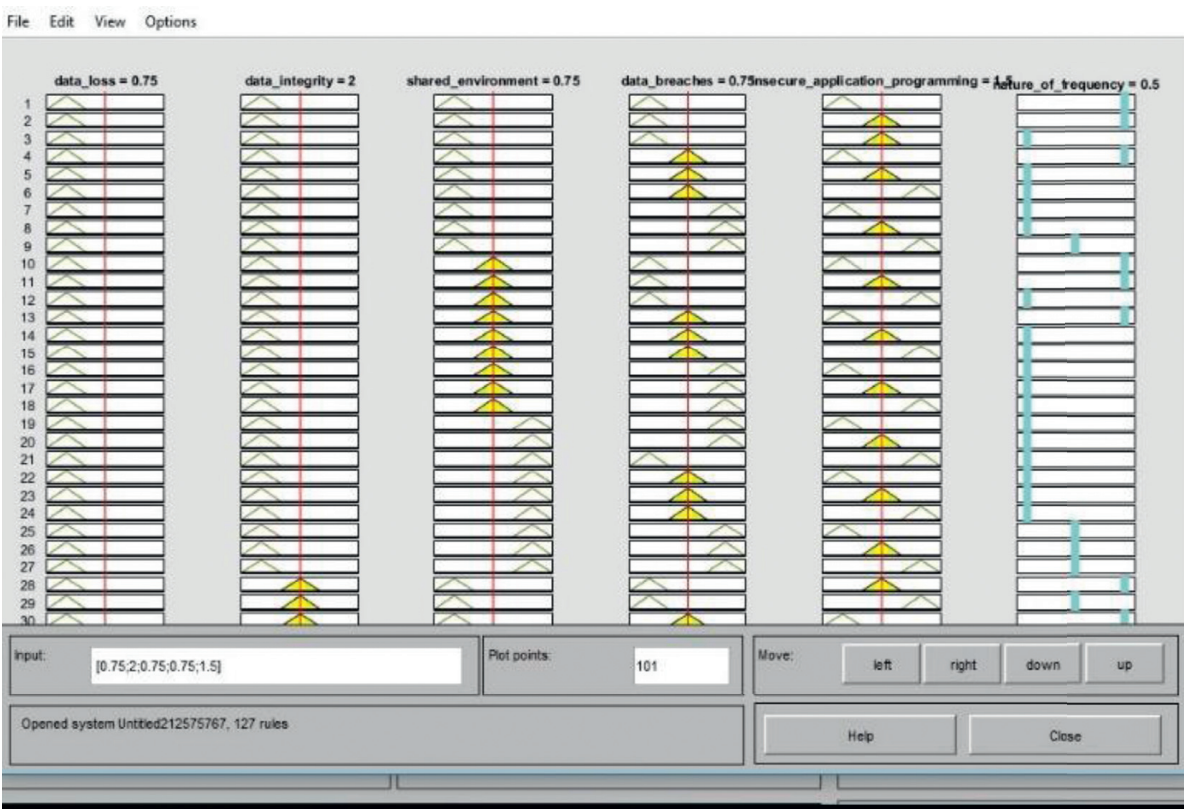

FIgURe 14: Fuzzy rule base viewer.

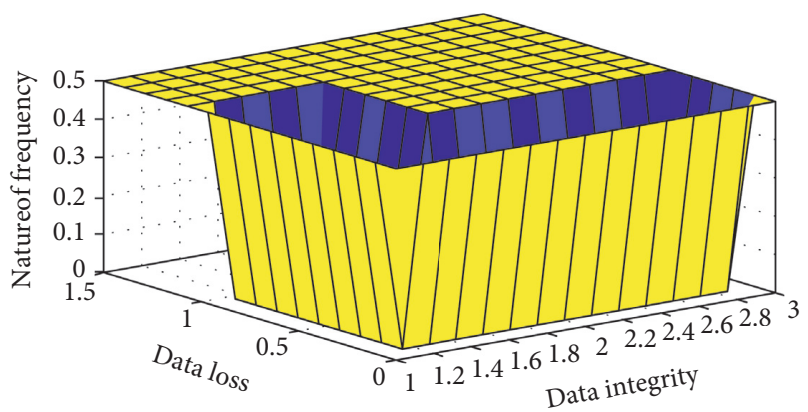

FIgURE 15: Surface plot of the system.

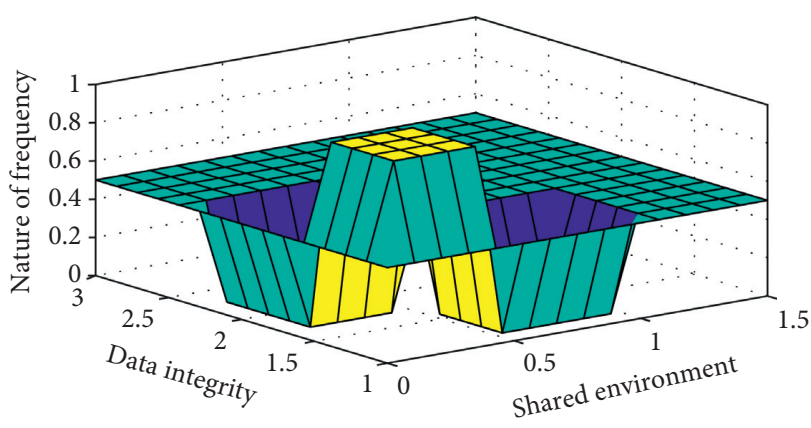

FIgURE 16: Surface plot of the system. 


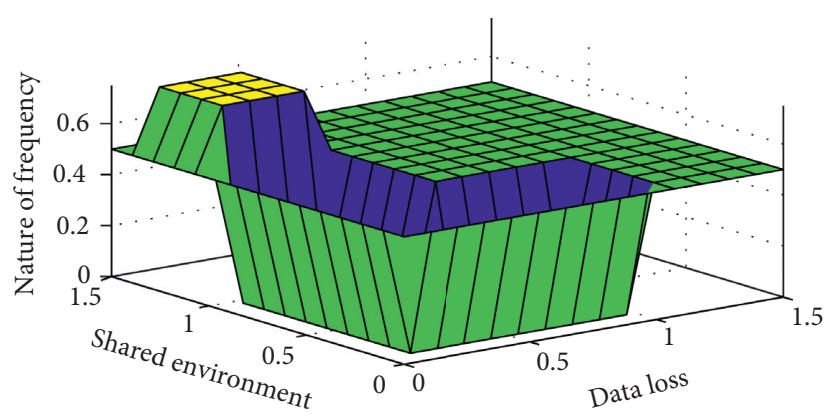

Figure 17: Surface plot of the system.

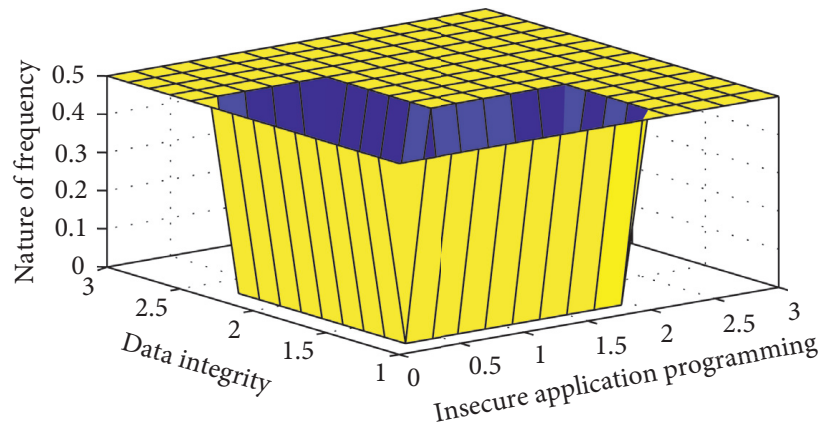

Figure 18: Surface plot of the system.

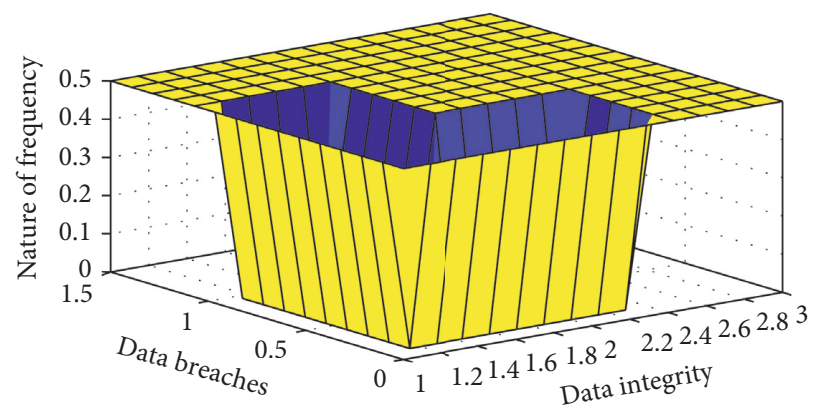

Figure 19: Surface plot of the system.

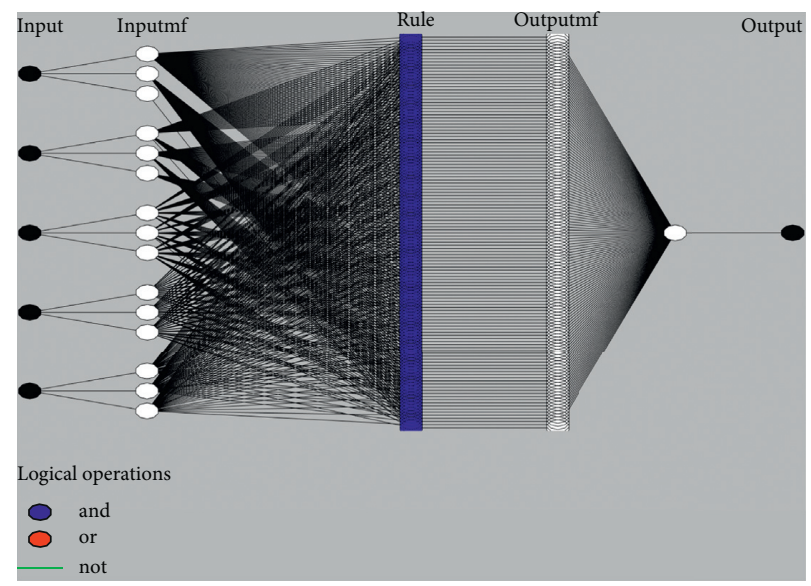

Figure 20: Neuro-fuzzy structure. 


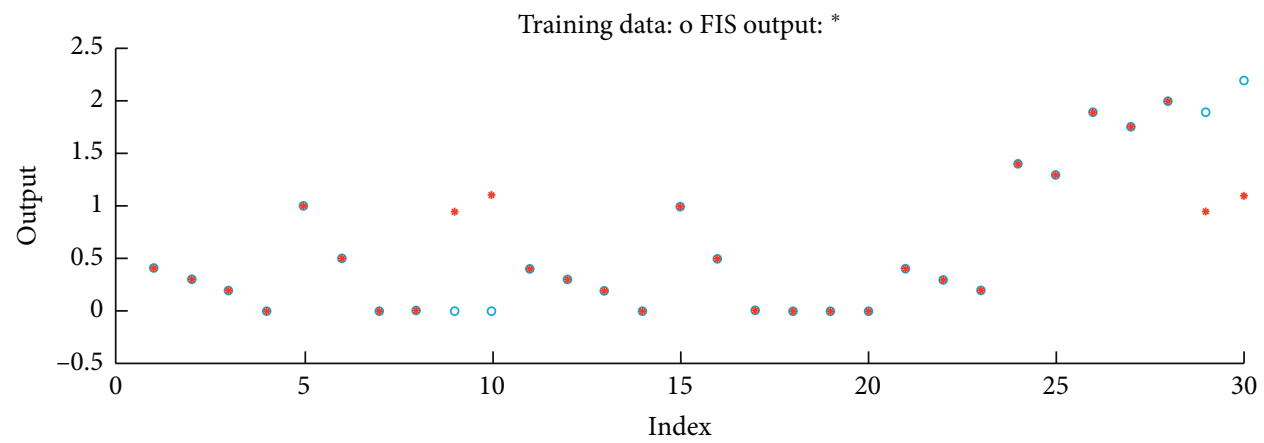

FIgURE 21: Training performance.

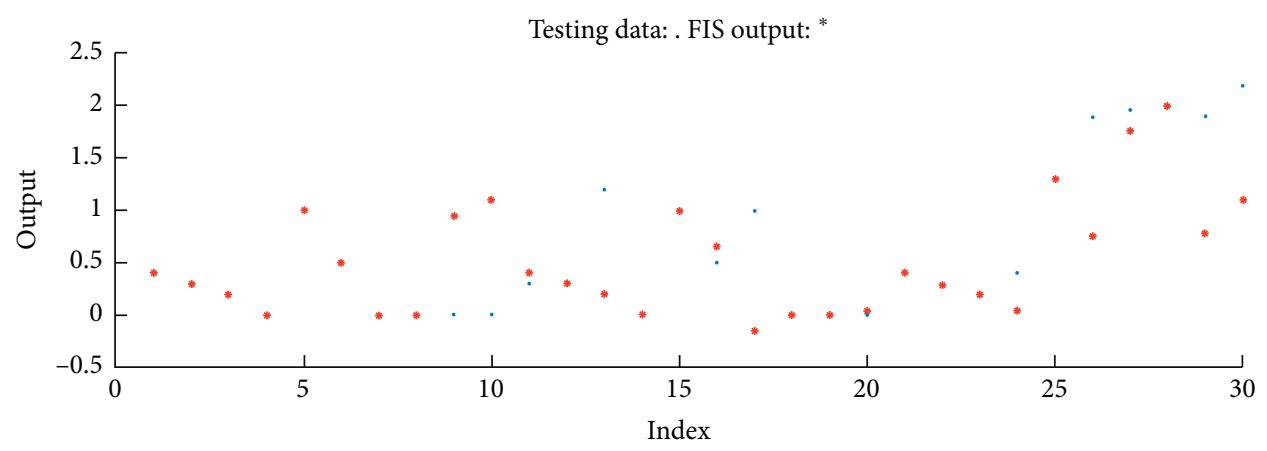

FIGURE 22: Testing performance.

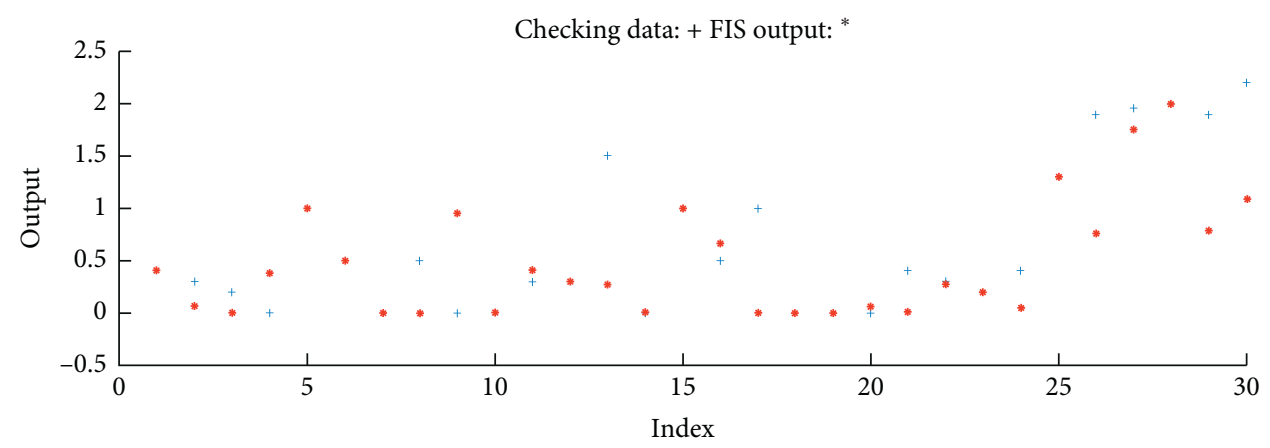

FIGURE 23: Checking performance.

TABLE 4: Inputs and output and their membership function.

\begin{tabular}{|c|c|}
\hline [Input 1$]=[$ data loss $]$ & $\begin{array}{c}\text { Range }=\left[\begin{array}{ll}0 & 3\end{array}\right], \text { num MFs }=3 \\
\text { MF } 1=\text { "no (data loss)": "trimf," }\left[\begin{array}{lll}0 & 0.5 & 1\end{array}\right] \\
\text { MF } 2 \text { = "medium (data loss)": "trimf," }\left[\begin{array}{lll}1 & 1.5 & 2\end{array}\right] \\
\text { MF } 3 \text { = "full (data loss)": "trimf," }\left[\begin{array}{lll}2 & 2.5 & 3\end{array}\right]\end{array}$ \\
\hline [Input 2] $=[$ data integrity $]$ & 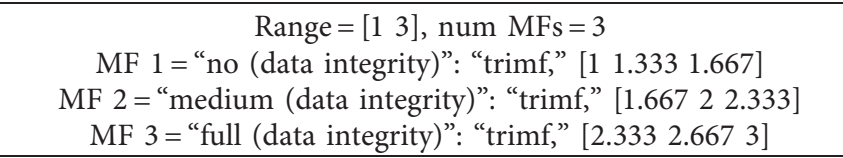 \\
\hline [Input 3] $=[$ shared environment $]$ & $\begin{array}{c}\text { Range }=\left[\begin{array}{ll}1 & 3\end{array}\right], \text { num MFs }=3 \\
\text { MF 1= "no (shared environment)": "trimf," }\left[\begin{array}{lll}1 & 1.333 & 1.667\end{array}\right] \\
\left.\text { MF } 2 \text { = "medium (shared environment)": "trimf," } \begin{array}{llll}1.667 & 2 & 2.333\end{array}\right] \\
\left.\text { MF 3 = "full (shared environment)": "trimf," [ } \begin{array}{lll}2.333 & 2.667 & 3\end{array}\right]\end{array}$ \\
\hline
\end{tabular}


TABLE 4: Continued.

\begin{tabular}{|c|c|}
\hline [Input 4$]=[$ data breaches $]$ & 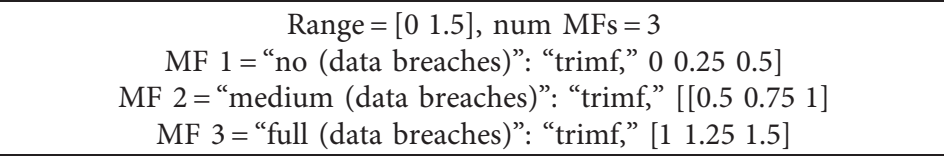 \\
\hline [Input 5] $=[$ insecure application programming $]$ & $\begin{array}{c}\text { Range }=\left[\begin{array}{ll}0 & 3\end{array}\right], \text { num MFs }=3 \\
\text { MF 1 = "no (insecure application programming)": "trimf," }\left[\begin{array}{lll}0 & 0.5 & 1\end{array}\right] \\
\text { MF } 2 \text { = "medium (insecure application programming)": "trimf," }\left[\begin{array}{lll}1 & 1.5 & 2\end{array}\right] \\
\text { MF 3 = "full (insecure application programming)": "trimf," }\left[\begin{array}{lll}2 & 2.5 & 3\end{array}\right]\end{array}$ \\
\hline [Output 1] $=[$ access control $]$ & $\begin{array}{c}\text { Range }=\left[\begin{array}{ll}0 & 1\end{array}\right], \text { num MFs }=3 \\
\text { MF } 1=\text { "no (access control)": "trimf," }\left[\begin{array}{lll}0 & 0.16 & .29\end{array}\right] \\
\text { MF 2 = "full (access control)": "trimf," }\left[\begin{array}{lll}0.6 & 0.85 & 1\end{array}\right] \\
\text { MF 3 = "medium (access control)": "trimf," }\left[\begin{array}{lll}0.29 & 0.45 & 0.6\end{array}\right]\end{array}$ \\
\hline
\end{tabular}

The main purpose of the proposed approach is to improve the accuracy and performance. The main contributions are given as follows:

(1) Fuzzy modeling is used to deal with uncertainty.

(2) The proposed approach is a new combination of different input parameters (data loss, data integrity, shared environment, data breaches, and insecure application programming), which is helpful to check the performance and easily understandable, which is an attribute that needs to be secured accordingly.

(3) The proposed approach using Sugeno-style Fuzzy Inference System techniques.

(4) The proposed model reaches high prediction and classification accuracy.

The output of Sugeno-type fuzzy expert system is either constant or linear. This is the reason that the results achieved in Sugeno-type fuzzy expert system are better.

\section{Conclusion}

In spite of the fact that distributed computing can enable organizations to do more by breaking the physical bonds between an IT foundation and its clients, raised security dangers must be defeated so as to profit completely from this new processing perspective that offers an imaginative arrangement of activity for relationship to grasp IT without frank hypothesis. Circulated processing is to sufficiently manage the security in cloud applications. The purpose of the study is to reduce security's obstacles that enable the global deployment and acceptance of portable distributed computing with the arrangement to highlight the security stresses that should be honestly tended to and made sense of how to comprehend the greatest limit of cloud preparing. In this article, we present a point-by-point examination of the cloud security issue. We evaluated the issue from the cloud building perspective. In setting of this examination, we find an undeniable detail of the cloud security issue and key features that should be affirmed by any proposed security methodology. The ANFIS decided the results as indicated by the base guideline and defuzzified the data. The fluffy control-based versatile framework enables the hubs in the system to accomplish a verified correspondence between those two targets. The outcomes got in a genuine ANFIS sending, in light of hubs, which does a fluffy-based control methodology to improve the security level while keeping correspondence unwavering quality and computational assets utilization among limits. Organizations these days are looking for creative approaches to develop and achieve their business objectives. With the assistance of distributed computing, this business will continue developing in the future. The eventual fate of distributed computing is splendid and will give advantages to both the host and the client.

\section{Data Availability}

The proposed scheme data used to support the findings of this study are available from the corresponding author on request.

\section{Conflicts of Interest}

The authors declare that they have no conflicts of interest.

\section{References}

[1] R. Soni, S. Ambalkar, and P. Bansal, "Security and privacy in cloud computing," in Proceedings of the 2016 Symposium on Colossal Data Analysis and Networking (CDAN), pp. 1-6, Indore, India, March 2016.

[2] E. Veldman and R. A. Verzijlbergh, "Distribution grid impacts of smart electric vehicle charging from different perspectives," IEEE Transactions on Smart Grid, vol. 6, no. 1, pp. 333-342, 2015.

[3] A. El-Yahyaoui and M. Dafir, "Data privacy in cloud computing," in Proceedings of the 2018 4th International Conference on Computer and Technology Applications (ICCTA), pp. 25-28, Istanbul, Turkey, May 2019.

[4] S. Maharjan, Q. Zhu, Y. Zhang, S. Gjessing, and T. Basar, "Dependable demand response management in the smart grid: a stackelberg game approach," IEEE Transactions on Smart Grid, vol. 4, no. 1, pp. 120-132, 2013.

[5] A. Panigrahi and M. R. Patra, "Network intrusion detection model based on fuzzy-rough classifiers," Handbook of Neural Computation, Elsevier, Amsterdam, Netherlands, pp. 109152, 2017.

[6] P. Wang, J. Ma, and L. Song, "Balanced interest distribution in smart grid: a Nash bargaining demand side management scheme," in Proceedings of the 2016 IEEE Global 
Communications Conference (GLOBECOM), Washington, DC, USA, December 2016.

[7] P. D. Diamantoulakis, K. N. Pappi, P.-Y. Kong, and G. K. Karagiannidis, "Game theoretic approach to demand side management in smart grid with user dependent acceptance prices," in Proceedings of the 2016 IEEE 84th Vehicular Technology Conference (VTC-Fall), Montreal, Canada, September 2016.

[8] T. Devi and R. Ganesan, "Environmental benefits of enhanced Hecc-elgamal cryptosystem for security in cloud data storage using soft computing techniques," International Journal of Electronics and Telecommunications, vol. 10, no. 5, pp. 115124, 2019.

[9] M. M. Mowla, I. Ahmad, D. Habibi, and Q. Viet Phung, "A green communication model for $5 \mathrm{G}$ systems," IEEE Transactions on Green Communications and Networking, vol. 1, no. 3, pp. 264-280, 2017.

[10] M. H. Alsharif, R. Nordin, N. F. Abdullah, and A. H. Kelechi, "How to make key $5 \mathrm{G}$ wireless technologies environmental friendly: a review," Transactions on Emerging Telecommunications Technologies, vol. 29, no. 1, Article ID e3254, 2017.

[11] K. Wang and L. He M. Gao, "Probabilistic model checking and scheduling implementation of energy router system in energy internet for green cities," IEEE Transactions on Industrial Informatics, vol. 14, no. 4, pp. 1501-1510, 2018.

[12] P. Liu, S. R. Chaudhry, T. Huang, X. Wang, and M. Collier, "Multi-factorial energy aware resource management in edge networks," IEEE Transactions on Green Communications and Networking, vol. 3, no. 1, pp. 45-56, 2018.

[13] M. Imran Tariq, "Agent based information security framework for hybrid cloud computing," KSII Transactions on Internet and Information Systems, vol. 13, no. 1, 2019.

[14] E. M. Mohamed, H. S. Abdelkader, and S. El-Etriby, "Data security model for cloud computing," Journal of Communication and Computer, vol. 10, no. 8, pp. 1047-1062, 2013.

[15] E. Cayirci, A. Garaga, A. S. de Oliveira, and Y. Roudier, "A risk assessment model for selecting cloud service providers," Journal of Cloud Computing: Advances, Systems and Applications, vol. 5, no. 1, 2016.

[16] Y. Wang, X. Hu, Y. Sun, D. J. Deng, A. Vinel, and Y. Z. K. Wang, "Wireless big data computing in smart grid," IEEE Wireless Communication, vol. 24, no. 2, pp. 58-64, 2017.

[17] K. Wang, X. Hu, H. Li, P. Li, D. Zeng, and S. Guo, "A survey on energy Internet communications for sustainability," IEEE Transactions on Sustainable Computing, vol. 2, no. 3, pp. 231-254, 2017.

[18] K. Wang, H. Li, Y. Feng, and G. Tian, "Big data analytics for system stability evaluation strategy in the energy Internet," IEEE Transactions on Industrial Informatics, vol. 13, no. 4, pp. 1969-1978, 2017.

[19] G. Anandini and M. G. I. Gupta, "An hour wise device scheduling approach for demand side management in smart grid using particle swarm optimization," in Proceedings of the 2016 National Power Systems Conference (NPSC), Bhubaneswar, India, December 2016.

[20] I. Gaied, F. Jemili, and O. G. Korbaa, "Intrusion detection based on neuro-fuzzy classification," in Proceedings of the 2015 IEEE/ACS 12th International Conference of Computer Systems and Applications (AICCSA), Marrakech, Morocco, November 2015.

[21] F. Ye, Y. Qian, and R. Q. Hu, "A real-time information based demand-side management system in smart grid," IEEE Transactions on Parallel and Distributed Systems, vol. 27, no. 2, pp. 329-339, 2016.
[22] N. M. Almutairy, K. H. A. Al-Shqeerat, and H. A. Al Hamad, "A taxonomy of virtualization security issues in cloud computing environments," Indian Journal of Science and Technssology, vol. 12, no. 3, pp. 1-19, 2019.

[23] F. A. Aoudia, M. Gautier, M. Magno, O. Berder, and L. A. Benini, "A generic framework for modeling MAC protocols in wireless sensor networks," IEEE/ACM Transactions on Networking, vol. 25, no. 3, pp. 1489-1500, 2017.

[24] P. Deb, D. De, and A. Mukherjee, "Natural computing in mobile network optimization," in Handbook of Research on Natural Computing for Optimization Problem, IGI Global, Hershey, PA, USA, 2016.

[25] M. H. Alsharif, "Techno-economic evaluation of a stand-alone power system based on solar power/batteries for global system for mobile communications base stations," Energies, vol. 10, no. 3, p. 392, 2017.

[26] M. Alsharif, "A solar energy solution for sustainable third generation mobile networks," Energies, vol. 10, no. 4, p. 429, 2017.

[27] F. Alshahwan, "Adaptive security framework in internet of things (IoT) for providing mobile cloud computing," in Mobile Computing-Technology and Applications, IntechOpen, London, UK, 2018. 